\title{
Um Mapeamento Sistemático de Gamificação em Software Educativo no Contexto da Comunidade Brasileira de Informática na Educação
}

\author{
Mariana Maia Peixoto, Carla Silva, Enyo Gonçalves, Jéssyka Vilela \\ Centro de Informática - Universidade Federal de Pernambuco (UFPE) \\ Av. Jornalista Aníbal Fernandes 50740-560 - Recife - PE - Brasil \\ $\{$ mmp2, ctlls, ejtg,jffv\}@cin.ufpe.br
}

\begin{abstract}
Gamification has been widely used in teaching as a support tool and motivation through game mechanics. It is a new area of research, but is showing results over the years with the Brazilian community of information technology in education. Therefore, it is necessary to analyze and consolidate their contributions in order to characterize them. This work aims to investigate and better understand how gamification helps in the teachinglearning process and identify to what extent is applied to this field. Therefore, this article presents a systematic mapping of using gamification in educational software in the context of Brazilian research presented at national events.
\end{abstract}

\begin{abstract}
Resumo. A gamificação vem sendo bastante utilizada no ensino como ferramenta de apoio e motivação através de mecânicas de games. Por ser um área de pesquisa recente, mas que vem apresentando resultados ao longo dos anos junto a comunidade brasileira de informática na educação é necessário analisar e consolidar suas contribuições de modo a caracterizá-las. Este trabalho busca investigar e entender melhor como gamificação contribui no processo ensino-aprendizagem bem como identificar em que medida é aplicado a este campo. Assim, este artigo apresenta um mapeamento sistemático do uso de gamificação em softwares educativos no contexto das pesquisas brasileiras apresentadas em eventos nacionais.
\end{abstract}

\section{Introdução}

Softwares vem cada vez mais sendo utilizados na área de educação como ferramenta facilitadora do processo de ensino aprendizagem. Nesse contexto, o software utilizado para atingir objetivos educacionais é denominado Software Educacional (SE) [Lovis 2007]. Existem quatro parâmetros que diferenciam um software qualquer de um educacional: fundamentação pedagógica, conteúdo, interação e a programação [Gomes e Padovani 2005]. Para Valente (1997), os SEs podem ser classificados de acordo com seus objetivos pedagógicos em: tutoriais, aplicativos, programação, exercícios e prática, multimídia e internet, simulação, modelagem e games.

Nesse contexto, é observado uma crescente utilização de técnicas de gamificação, em SEs, para apoiar a aprendizagem. A gamificação (do inglês, gamification) corresponde ao uso de mecanismos de games orientados ao objetivo de resolver problemas práticos ou de despertar engajamento entre um público específico [Viana et al. 2013]. Assim, a gamificação não implica em criar um game que aborde o problema, recriando a situação dentro de um mundo virtual. Ela envolve usar as mesmas 
estratégias, métodos e pensamentos utilizados para resolver problemas nos mundos virtuais (games) em situações do mundo real [Fardo 2013].

A finalidade da gamificação dentro de um ambiente educacional é envolver os usuários no contexto da aprendizagem, permitindo-lhes dominar o material estudado [Morrison e Disalvo 2014]. Uma das vantagens de sua aplicação na educação é proporcionar um sistema em que os alunos consigam visualizar o efeito de suas ações e aprendizagens, na medida em que o avanço das atividades ocorre. Desta forma fica mais fácil compreender a relação das partes com o todo, como acontece nos games [Fardo 2013].

No entanto, a comunidade de informática na educação ainda carece de uma compreensão abrangente de como a gamificação é utilizada em SEs. Assim, o principal objetivo deste trabalho é investigar e entender melhor como gamificação contribui no processo ensino-aprendizagem, bem como identificar em que medida é aplicado a este campo. A fim de cumprir o nosso objetivo, foi realizado um mapeamento sistemático da literatura para investigar direcionamentos da utilização da gamificação para apoiar a educação no contexto brasileiro apresentados em eventos nacionais de informática na educação, seguindo um protocolo de avaliação pré-definido.

O trabalho está organizado da seguinte maneira. A Seção 2 discute alguns trabalhos relacionados. Na Seção 3, o método de pesquisa utilizado é apresentado, incluindo o desenho de pesquisa, limitações e ameaças à validade. Na Seção 4, são expostos os resultados. E por ultimo, a Seção 5 apresenta as conclusões e os direcionamentos para trabalhos futuros.

\section{Trabalhos Relacionados}

Borges et al. (2013) realizaram um mapeamento sistemático de literatura, com 26 artigos selecionados, para investigar a gamificação aplicada à educação. Como resultado um mapa das pesquisas na área foi desenvolvido e os tópicos mais (e menos) investigados foram identificados, apontando que a maioria dos estudos concentra-se em investigar como a gamificação pode ser utilizada na área de ensino para motivar estudantes, aprimorar suas habilidades e maximizar o aprendizado.

Diana et al. (2014) realizaram uma revisão sistemática de literatura para identificar as possíveis relações entre as características da gamificação e os elementos que compõem a Teoria Flow. A Teoria Flow aponta características que as pessoas apresentam ao desenvolver uma atividade prazerosa, resultando na felicidade e bem estar. Como resultados, o trabalho apresenta 39 características da gamificação que podem ser utilizadas para estabelecer a relação com a Teoria do Flow.

O trabalho de Peixoto e Silva (2015) apresenta uma revisão sistemática de literatura com intuito de investigar características de gamificação para ambientes educacionais e, assim, definir um conjunto de requisitos para serem reusados no desenvolvimento de softwares educacionais gamificados. Como resultado do estudo, foram reportados os requisitos descobertos, os tipos das pesquisas realizadas, os anos de publicação e os países dessas pesquisas referentes a ambientes gamificados voltados à educação. E por último, foi observado que ainda não há consenso sobre um conjunto de características que devem existir em SEs gamificados. 
É importante salientar que os trabalhos discutidos nesta seção não foram desenvolvidos apenas no contexto brasileiro. Identificar, analisar e quantificar resultados de pesquisas brasileiras neste rumo constitui tarefa importante ser considerada para dimensionar o uso de gamificação no ensino brasileiro e as características inerentes ao uso deste técnica no país. A próxima seção descreve o protocolo de avaliação utilizado neste trabalho.

\section{Método: Protocolo de Pesquisa}

O presente mapeamento sistemático de literatura seguiu os procedimentos indicados por Kitchenham e Charters (2007). Sendo assim, os seguintes estágios foram utilizados:

Estágio 1 - Planejamento: Identificação da necessidade do mapeamento; Especificação da (s) pergunta (s) de pesquisa; e Desenvolvimento de um protocolo de mapeamento.

Estágio 2 - Realização: Identificação de pesquisa; Seleção de estudos primários; Extração de dados; e Síntese de dados.

Estágio 3 - Relato: Especificação dos mecanismos de divulgação; e Formatação do relatório principal.

\subsection{Questões de Pesquisa}

Especificar as questões de pesquisa é a parte mais importante de qualquer mapeamento sistemático [Kitchenham e Charters 2007]. Portanto, esta pesquisa responderá as seguintes questões:

QP1: Quais são os anos de publicação e instituições que investigam o uso de softwares gamificados voltados à educação?

QP2: Quais são as modalidades de ensino os tipos de pesquisa e tipos de softwares publicados referentes a gamificação voltada à educação?

QP3: Quais são os objetivos educacionais inerentes à criação de softwares gamificados?

QP4: Quais são as principais características necessárias à criação de softwares gamificados voltados à educação?

\subsection{Processo de Busca}

O rigor da processo de busca é um fator que distingue mapeamentos sistemáticos de outros tipos de revisões [Kitchenham e Charters 2007]. A estratégia para a identificação das pesquisas publicadas implica na definição das fontes de busca, da linguagem dos estudos e das strings de busca. Foram realizadas buscas nas fontes apresentadas na Tabela 1.

Tabela 1. Fontes de Busca

\begin{tabular}{|c|c|}
\hline Fonte de Busca & Site \\
\hline $\begin{array}{l}\text { Revista Novas Tecnologias } \\
\text { na Educação (RENOTE) }\end{array}$ & http://seer.ufrgs.br/RENOTE \\
\hline $\begin{array}{l}\text { Revista Brasileira de } \\
\text { Informática na Educação } \\
\text { (RBIE) }\end{array}$ & http://www.br-ie.org/pub/index.php/rbie \\
\hline
\end{tabular}


CBIE-LACLO 2015

Anais do XXI Workshop de Informática na Escola (WIE 2015)

\begin{tabular}{|c|c|}
\hline $\begin{array}{l}\text { Revista Informática na } \\
\text { educação: teoria \& prática }\end{array}$ & http://seer.ufrgs.br/InfEducTeoriaPratica/; \\
\hline $\begin{array}{l}\text { Revista Brasileira de } \\
\text { Aprendizagem Aberta e a } \\
\text { Distância (RBAAD) }\end{array}$ & http://www.abed.org.br/revistacientifica/_brazilian/ \\
\hline $\begin{array}{l}\text { Simpósio Brasileiro de } \\
\text { Informática na Educação } \\
\text { (SBIE) }\end{array}$ & http://www.br-ie.org/pub/index.php/sbie/index \\
\hline $\begin{array}{l}\text { Workshop de Informática na } \\
\text { Escola (WIE) }\end{array}$ & http://www.br-ie.org/pub/index.php/wie/issue/archive \\
\hline $\begin{array}{l}\text { Workshop sobre Educação } \\
\text { em Computação (WEI) }\end{array}$ & http://csbc2014.cic.unb.br/index.php/wei \\
\hline
\end{tabular}

As fontes de busca, aqui escolhidas, justificam-se por serem fontes relevantes para a área de informática na educação brasileira. As linguagens adotadas foram Inglês, Português e Espanhol. A busca ocorreu de forma manual, entretanto para seguir o rigor necessário para a identificação das pesquisas, em um mapeamento sistemático de literatura, a seguinte string de busca, contendo sinônimos relevantes, foi desenvolvida:

String de Busca:

("Gamification" OR "Gamifies" OR "Gamifying" OR "Gamified") AND ("Educational" OR "Learning" OR "Educative") AND ("Type" OR "Characteristics" OR "Modality" OR "Objective" OR "Software Engineering" OR "Software")

\subsection{Seleção dos Estudos Primários}

Uma vez que os estudos primários potencialmente relevantes tenham sido obtidos, eles precisam ser avaliados, para isso é necessário indicar alguns critérios de inclusão e exclusão. Estes critérios destinam-se a identificar os estudos primários que fornecem evidência direta sobre a questão de pesquisa [Kitchenham e Charters 2007].

Para obter resultados coerentes os critérios de inclusão e exclusão foram definidos com base nas questões de pesquisa e são apresentados na Tabela 2.

Tabela 2. Critérios de inclusão e exclusão

Critérios de Inclusão Critérios de Exclusão

Estudos que analisam características e Estudos realizados antes de 2010

teorias de aprendizagem de ambientes

educacionais gamificados

Estudos que relatam e descrevem o Estudos duplicados desenvolvimento de ambientes

educacionais gamificados

Estudos acessíveis na rede da universidade Estudos secundários e terciários

Estudos originais nas línguas: Inglês, Estudos que tratam a gamificação na Português e Espanhol educação como trabalhos futuros

O processo de seleção contou com duas etapas distintas:

- Etapa 1: Leitura dos títulos, palavras-chave, resumo e conclusão. Considerando os critérios de inclusão e exclusão. 
- Etapa 2: Os estudos incluídos são documentados. Cada estudo é lido por completo e através de formulários a extração dos dados é realizada.

\subsection{Ameaças à Validade}

O protocolo de mapeamento seguiu alguns passos para garantir que a pesquisa seja a mais correta e objetiva possível. Porém, foram identificadas possíveis limitações em alguns momentos.

O primeiro momento refere-se à identificação dos artigos publicados, a string de busca utilizada pode não contemplar todos os sinônimos existentes para o termo "Software Educacional Gamificado" e, assim, ser insuficiente para capturar todos os estudos da área.

O segundo momento refere-se ao processo de busca. As buscas foram realizadas em eventos e revistas nacionais. No entanto, é possível que vários estudos tenham sido publicados internacionalmente e assim não refletir todas as pesquisas desenvolvidas pela comunidade brasileira.

\section{Apresentação dos Resultados e Discussão}

Nesta seção são apresentados os resultados do mapeamento sistemático realizado e uma discussão relativa a estes. Isto envolve a apresentação do processo de seleção e extração de dados e apresentação das respostas às questões de pesquisa levantadas.

\subsection{Processo de Seleção e Extração de Dados}

O processo de seleção dos trabalhos foi realizado em duas etapas. A primeira etapa pode ser visualizada na Tabela 3. Para esta etapa foram considerados: leitura do título, palavras-chave, resumo e conclusão e posteriormente foram excluídos trabalhos irrelevantes para as questões investigadas.

Tabela 3. Quantitativo dos Resultados de Busca da Etapa 1

\begin{tabular}{|c|c|c|c|}
\hline Fontes de Busca & $\begin{array}{l}\text { Resultados da } \\
\text { Busca }\end{array}$ & Excluídos & $\begin{array}{c}\text { Estudos } \\
\text { Selecionados }\end{array}$ \\
\hline 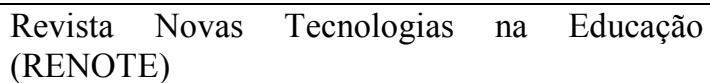 & 6 & 2 & 4 \\
\hline $\begin{array}{l}\text { Revista Brasileira de Informática na Educação } \\
\text { (RBIE) }\end{array}$ & 0 & 0 & 0 \\
\hline Revista Informática na educação: teoria \& prática & 0 & 0 & 0 \\
\hline $\begin{array}{l}\text { Revista Brasileira de Aprendizagem Aberta e a } \\
\text { Distância (RBAAD) }\end{array}$ & 0 & 0 & 0 \\
\hline $\begin{array}{l}\text { Simpósio Brasileiro de Informática na Educação } \\
\text { (SBIE) }\end{array}$ & 11 & 2 & 9 \\
\hline Workshop de Informática na Escola (WIE) & 0 & 0 & 0 \\
\hline $\begin{array}{l}\text { Workshop sobre Educação em Computação } \\
\text { (WEI) }\end{array}$ & 2 & 1 & 1 \\
\hline Total & 19 & 5 & 14 \\
\hline
\end{tabular}

Dos dezenove artigos encontrados, com o auxílio da string de busca, cinco foram excluídos por não responder nenhumas das questões de pesquisa. A realização da segunda etapa, cujos resultados são listados na Tabela 4, os estudos incluídos na etapa anterior foram lidos, e por meio do formulário, a extração dos dados foi realizada. $\mathrm{Na}$ Tabela 4 é possível observar uma breve descrição de cada trabalho selecionado. 
CBIE-LACLO 2015

Anais do XXI Workshop de Informática na Escola (WIE 2015)

Tabela 4. Quantitativo dos Resultados de Busca da Etapa 2

\begin{tabular}{|c|c|c|}
\hline & & Etapa 2 \\
\hline Fontes de Busca & Referência & Breve Descrição \\
\hline \multirow{4}{*}{$\begin{array}{l}\text { Revista Novas } \\
\text { Tecnologias na } \\
\text { Educação } \\
\text { (RENOTE) }\end{array}$} & Fardo (2013) & Conceitua a gamificação para a educação \\
\hline & $\begin{array}{l}\text { Klock et al. } \\
\quad(2014)\end{array}$ & $\begin{array}{l}\text { Analisa técnicas de gamificação em ambientes virtuais de } \\
\text { aprendizagem. }\end{array}$ \\
\hline & $\begin{array}{l}\text { Paschoal et al. } \\
\text { (2014) }\end{array}$ & $\begin{array}{l}\text { Apresenta o desenvolvimento de um aplicativo gamificado como } \\
\text { apoio ao ensino de informática a um grupo de idosos }\end{array}$ \\
\hline & $\begin{array}{l}\text { Bissolotti et } \\
\text { al. (2014) }\end{array}$ & $\begin{array}{l}\text { Discute como a gamificação pode potencializar os processos de } \\
\text { ensino e aprendizagem }\end{array}$ \\
\hline \multirow{9}{*}{$\begin{array}{l}\text { Simpósio } \\
\text { Brasileiro de } \\
\text { Informática na } \\
\text { Educação (SBIE) }\end{array}$} & $\begin{array}{l}\text { Fernandes e } \\
\text { Castro (2013) }\end{array}$ & Apresenta um ambiente de ensino de química orgânica gamificado \\
\hline & $\begin{array}{l}\text { França e } \\
\text { Reategui } \\
(2013)\end{array}$ & $\begin{array}{l}\text { Apresenta um estudo sobre o uso dos conceitos de gamificação em } \\
\text { um ambiente de aprendizagem baseado em questionamento. }\end{array}$ \\
\hline & $\begin{array}{c}\text { Andrade e } \\
\text { Canese (2013) }\end{array}$ & $\begin{array}{l}\text { Apresenta um sistema web gamificado para a aprendizagem de } \\
\text { lógica formal }\end{array}$ \\
\hline & $\begin{array}{l}\text { Ellwanger et } \\
\text { al. (2014) }\end{array}$ & $\begin{array}{l}\text { Apresenta as relações entre gamificação, padrões de interface e } \\
\text { mobilidade no desenvolvimento de aplicações educacionais }\end{array}$ \\
\hline & $\begin{array}{l}\text { Alves et al. } \\
\quad(2014)\end{array}$ & $\begin{array}{l}\text { Apresenta o uso da gamificação para o auxílio ao ensino de } \\
\text { ecologia }\end{array}$ \\
\hline & $\begin{array}{l}\text { Toda et al. } \\
\quad(2014)\end{array}$ & $\begin{array}{l}\text { Apresenta o desenvolvimento de uma aplicação web gamificada } \\
\text { para auxiliar no ensino da matemática para alunos do ensino } \\
\text { fundamental }\end{array}$ \\
\hline & $\begin{array}{l}\text { Falcão et al. } \\
\qquad(2014)\end{array}$ & $\begin{array}{l}\text { Apresenta uma ferramenta de apoio ao ensino presencial utilizando } \\
\text { gamificação e design de jogos }\end{array}$ \\
\hline & $\begin{array}{l}\text { Seixas et al. } \\
\qquad(2014)\end{array}$ & $\begin{array}{l}\text { Discute a gamificação como estratégia no engajamento de } \\
\text { estudantes do ensino fundamental }\end{array}$ \\
\hline & $\begin{array}{l}\text { Medeiros et } \\
\text { al. }(2014)\end{array}$ & $\begin{array}{l}\text { Apresenta uma abordagem gamificada para prevenção do uso de } \\
\text { drogas com adolescentes }\end{array}$ \\
\hline $\begin{array}{lr}\text { Workshop } & \text { sobre } \\
\text { Educação } & \text { em } \\
\text { Computação } & \\
\text { (WEI) } & \end{array}$ & $\begin{array}{l}\text { Jucá et al. } \\
\text { (2014) }\end{array}$ & $\begin{array}{l}\text { Apresenta uma aplicação da gamificação na disciplina de } \\
\text { empreendedorismo }\end{array}$ \\
\hline
\end{tabular}

\subsection{Resposta às Questões de Pesquisa}

A primeira questão de pesquisa tem como objetivo realizar o levantamento da distribuição temporal dos trabalhos.

A busca pretendia encontrar trabalhos entre os anos de 2010 à 2015, porém só foram selecionados nos anos de 2013 e 2014. Na Tabela 5 é possível visualizar que 2014 é o ano com o maior número de publicações 71,4\%. Esta constatação pode ser justificada pelo fato de que a gamificação é um conceito recente.

Observando o somatório de todos os anos pesquisados, a fonte de busca SBIE destaca-se com o maior número de artigos selecionados $9(64,2 \%)$ trabalhos. A fonte WEI possui o menor resultado com 1 artigo (7,14\%).

Tabela 5: Anos de Publicação

\begin{tabular}{|c|c|c|c|c|c|c|}
\hline \multirow{3}{*}{ Fontes de Busca } & \multicolumn{6}{|c|}{ Ano } \\
\hline & \multicolumn{2}{|c|}{2013} & \multicolumn{2}{|c|}{2014} & \multicolumn{2}{|c|}{ Total } \\
\hline & $\mathrm{f}^{*}$ & $\%$ & $f^{*}$ & $\%$ & $f^{*}$ & $\%$ \\
\hline RENOTE & 1 & 25,0 & 3 & 75,0 & 4 & 28,5 \\
\hline SBIE & 3 & 33,3 & 6 & 66,6 & 9 & 64,2 \\
\hline
\end{tabular}


CBIE-LACLO 2015

Anais do XXI Workshop de Informática na Escola (WIE 2015)

\begin{tabular}{lcccccc}
\hline WEI & 0 & 0 & 1 & 100,0 & 1 & 7,14 \\
\hline Total & 4 & 28,5 & 10 & 71,4 & 14 & 100,0 \\
\hline Nota:* Frequência. & & & & & &
\end{tabular}

$\mathrm{Na}$ Tabela 6 é possível observar que foram encontradas treze instituições de ensino de sete estados brasileiros, sendo a Universidade Tecnológica Federal do Paraná a única com duas publicações e o estado com o maior número de artigos foi Rio Grande do Sul (4), seguido por Santa Catarina (3).

\begin{tabular}{|c|c|c|c|}
\hline $\begin{array}{c}\text { Fonte de } \\
\text { Busca }\end{array}$ & Instituição & Estado & $\mathrm{N}^{*}$ \\
\hline \multirow[t]{4}{*}{ RENOTE } & Universidade de Caxias do Sul & $\begin{array}{l}\text { Rio Grande do } \\
\text { Sul }\end{array}$ & 1 \\
\hline & Universidade do Estado de Santa Catarina & Santa Catarina & 1 \\
\hline & Universidade de Cruz Alta & $\begin{array}{l}\text { Rio Grande do } \\
\text { Sul }\end{array}$ & 1 \\
\hline & Universidade Federal de Santa Catarina & Santa Catarina & 1 \\
\hline \multirow[t]{8}{*}{ SBIE } & Universidade do Vale de Itajaí & Santa Catarina & 1 \\
\hline & Universidade Federal do Rio Grande do Sul & $\begin{array}{l}\text { Rio Grande do } \\
\text { Sul }\end{array}$ & 1 \\
\hline & $\begin{array}{l}\text { Instituto Federal de Educação, Ciência e } \\
\text { Tecnologia do Espírito Santo }\end{array}$ & Espírito Santo & 1 \\
\hline & $\begin{array}{l}\text { Universidade Regional Integrada do Alto } \\
\text { Uruguai e das Missões }\end{array}$ & $\begin{array}{l}\text { Rio Grande do } \\
\text { Sul }\end{array}$ & 1 \\
\hline & $\begin{array}{l}\text { Centro Federal de Educação Tecnológica } \\
\text { Celso Suckow da Fonseca }\end{array}$ & Rio de Janeiro & 1 \\
\hline & Universidade Estadual de Londrina & Paraná & 1 \\
\hline & Universidade Tecnológica Federal do Paraná & Paraná & 2 \\
\hline & Universidade Federal de Pernambuco & Pernambuco & 1 \\
\hline WEI & Universidade Federal do Ceará & Ceará & 1 \\
\hline
\end{tabular}

A segunda questão de pesquisa pretende identificar as modalidades de ensino, os tipos de pesquisa e tipos de softwares publicados referentes a gamificação voltada à educação.

As modalidades de ensino, dos trabalhos, podem ser observados no gráfico da Figura 1. Entre eles, metade, ou seja, sete trabalhos não especificaram para qual modalidade de ensino estavam se referindo. Terceira Idade e Ensino Médio foram referenciados por apenas um trabalho cada.

\section{Modalidade}

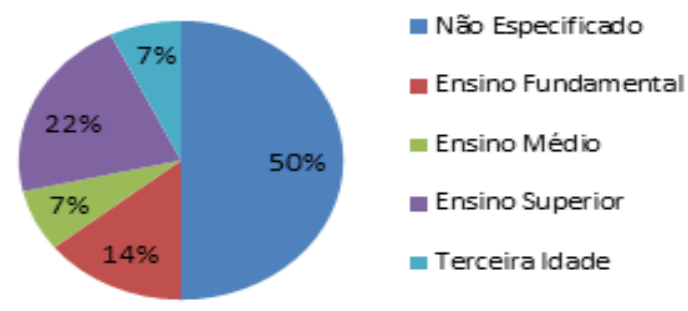

Figura 1. Modalidade Educacional 
A variável Tipo do Estudo foi classificada com base no relato dos próprios trabalhos. A Tabela 6 apresenta os quatro tipos de classificação. Desenvolvimento se destacou com 6 trabalhos, seguido por exemplo 4, proposta de metodologia 2 e metodologia empírica 2. Das modalidades de ensino que foram especificadas, a que obteve a maior variação de tipos de estudo foi o ensino superior.

Tabela 6 . Tipo de Estudo por Modalidade de Ensino

\begin{tabular}{cccccc}
\hline & \multicolumn{5}{c}{ Tipo de Estudo } \\
\cline { 2 - 6 } Modalidade & Desenvolvimento & Exemplo & $\begin{array}{c}\text { Metodologia } \\
\text { Empírica }\end{array}$ & $\begin{array}{c}\text { Proposta de } \\
\text { Metodologia }\end{array}$ & Total \\
\hline Terceira Idade & 0 & 0 & 1 & 0 & 1 \\
$\begin{array}{c}\text { Ensino } \\
\text { Fundamental } \\
\text { Ensino Médio }\end{array}$ & 1 & 0 & 1 & 0 & 2 \\
$\begin{array}{c}\text { Ensino } \\
\text { Superior } \\
\text { Não }\end{array}$ & 1 & 0 & 0 & 0 & 1 \\
Especificado & 3 & 1 & 0 & 1 & 3 \\
\hline Total & $6(43,0 \%)$ & $4(29,0 \%)$ & $2(12,3 \%)$ & $2(14,0 \%)$ & $14(100 \%)$ \\
\hline
\end{tabular}

Os trabalhos referentes à metodologia empírica discutiram e avaliaram o uso de técnicas e modelos de softwares educacionais gamificados. Os trabalhos que relataram o desenvolvimento de softwares educacionais gamificados apresentaram sistemas prontos, a forma de implementação, o design e alguns realizaram discussão sobre a experiência. Alguns artigos discutiram exemplos de softwares de terceiros para e assim apresentar uma visão geral e realizaram análises. Os trabalhos de propostas metodológicas apresentaram diretrizes e características.

Alguns trabalhos não foram precisos ao indicarem os tipos de softwares educacionais utilizados, entretanto foi possível destacar algumas modalidades e a quantidade de citações. São elas: Ambiente Virtual de Aprendizagem (1); Ambiente para Educação a Distância (1); Ambiente de Ensino (1); Ambiente de Aprendizagem Baseado em Questionamento (1); Game (3): Game para Dispositivo Móvel (1); Sistema Web (4); e Aplicativo para Dispositivo Móvel (1).

A terceira pergunta pretende averiguar os objetivos educacionais inerentes à criação de softwares gamificados.

Os objetivos educacionais e o número de citações encontrados foram: Engajar (7); Motivar (5); Apoiar o Ensino (2); Promover Interação (1); Estimular o Interesse (1); Reforçar a Aprendizagem (1); Gerar Novos Objetivos Educacionais (1); Recompensar (1); e Prevenir (1). É importante destacar que alguns trabalhos apresentaram mais de um objetivo educacional. Em seu trabalho, Borges et al. (2013) apresentam alguns objetivos educacionais alcançados com a gamificação, tais como: Aprimorar Habilidades; Desafios; Guidelines; Engajamento; Maximização do Aprendizado; Mudança de Comportamento; Socialização. Entretanto, apenas o objetivo engajamento se fez presente em ambos os estudos.

A quarta pergunta de pesquisa pretende investigar as principais características necessárias à criação de softwares gamificados voltados à educação. As principais características identificadas através dos estudos selecionados estão dispostas na Tabela 
7 abaixo. Por meio da análise realizada foi possível identificar que existem variações de nomenclatura para a mesma característica.

\begin{tabular}{|c|c|c|c|}
\hline Característica & Característica & Característica & Característica \\
\hline $\begin{array}{ll}\text { Pontos (Pontos } & \text { de } \\
\text { experiência, Pontos } \\
\text { resgatáveis, Pontos de } \\
\text { habilidade, Pontos } & \text { de } \\
\text { carma, Pontos } & \text { de } \\
\text { reputação, pontos } & \text { de } \\
\text { resolução) }\end{array}$ & $\begin{array}{l}\text { Níveis (Níveis de game, } \\
\text { Níveis de dificuldade, } \\
\text { Níveis de jogador) }\end{array}$ & $\begin{array}{l}\text { Rankings } \\
\text { não } \\
\text { Rankinging que } \\
\text { desincentiva, } \\
\text { Ranking } \\
\text { Ranking } \\
\text { Experiência) }\end{array}$ & $\begin{array}{l}\text { Tabelas de líderes } \\
\text { (Tabelas de líderes } \\
\text { globais, Tabelas de } \\
\text { líderes parciais) }\end{array}$ \\
\hline Medalhas & Status & Metas & Consultar estatísticas \\
\hline $\begin{array}{l}\text { Notificação de } \\
\text { progresso dos amigos }\end{array}$ & $\begin{array}{l}\text { Desafios, } \\
\text { desafios }\end{array}$ & $\begin{array}{l}\text { Elementos } \\
\text { colecionáveis }\end{array}$ & $\begin{array}{l}\text { Liberação de atividades } \\
\text { e conteúdos }\end{array}$ \\
\hline $\begin{array}{l}\text { Feedback, Feedback } \\
\text { constante }\end{array}$ & Novas funcionalidades & $\begin{array}{l}\text { Personalização, } \\
\text { Personagem, Avatar }\end{array}$ & $\begin{array}{l}\text { Listagem de problemas } \\
\text { resolvidos }\end{array}$ \\
\hline Trocas & Conquistas & Reputação & Evolução pessoal \\
\hline Missões & Presente & Estratégias & Títulos \\
\hline Narrativa & Integração & Liberar acesso & Desejos dos usuários \\
\hline Evolução & Loops & Itens bloqueados & Autoexpressão \\
\hline Grupos & Engajamento & Conquistar espaço & Altruísmo \\
\hline Tratamento de erro & Reforço & Ganhar visibilidade & Frequência de acesso \\
\hline $\begin{array}{l}\text { Diferentes } \\
\text { experimentações }\end{array}$ & Regras & $\begin{array}{l}\text { Históricos } \\
\text { conquistas }\end{array}$ & Oportunidades \\
\hline Ciclos rápidos & Nivelamento & Moeda virtual & Pressão Temporal \\
\hline Aumentar a dificuldade & Barra de progresso & Prêmios & Novidades \\
\hline Dividir tarefas & Recompensas & Dicas & Renovação \\
\hline Competição & Conflito & Revelação progressiva & Reconhecimento \\
\hline Colaboração & Fases & Obrigação social & Classificação Constante \\
\hline
\end{tabular}

Muitas características aqui encontrados fazem parte do MDA (Mechanics, Dynamics and Aesthetics) framework, que corresponde ao português mecânica, dinâmica e estética e representam os elementos básicos de gamificação, proposto por Kim e Lee (2013) que por sua vez é fundamentado na concepção de game design. Entretanto, este trabalho apresenta uma maior variedade de características que podem estar presentes em ambientes de aprendizagem gamificados.

Assim, foi constatado que muitas características apresentadas na Tabela 7 estão ligadas a conceitos preexistentes de design de games comuns e games de aprendizagem. Entretanto, ainda não existe consenso de um conjunto fixo de características que devem estar presentes ao realizar gamificação em uma plataforma de aprendizagem.

\section{Considerações Finais e Trabalhos Futuros}

Neste artigo foi realizado um mapeamento sistemático de literatura para investigar e entender melhor como gamificação contribui no processo ensino-aprendizagem bem como identificar em que medida é aplicado a este campo no contexto brasileiro.

Treze instituições brasileiras realizam estudos no campo da gamificação para a educação, as modalidades de ensino só foram apresentadas em metade dos trabalhos, apenas um estudo apresentou uma metodologia empírica para validação e o tipo de software mais utilizado é o sistema web. 
Os resultados sugerem que muitos trabalhos relatam características ligadas a conceitos de games e que não há consenso de um conjunto fixo de características que devem existir em SEs gamificados que os diferenciem de games educacionais.

Como trabalhos futuros, pretende-se ampliar o mapeamento na busca de estender esta abordagem para apoiar a criação de uma padronização que contemple as características de gamificação associadas aos estilos de aprendizagem dos alunos. A padronização será relevante para o estabelecimento um modelo de qualidade que sirva como referência para o desenvolvimento de software que atenda às necessidades dos alunos e professores.

\section{Referências}

Borges, S. B.; Durelli, V. H. S.; Reis, H. M.; Isotani, S. (2014). A systematic mapping on gamification applied to education. In Proceedings of the 29th Annual ACM Symposium on Applied Computing (SAC '14). ACM, New York, NY, USA, 216222.

Diana, J. B.; Golfetto, I. F.; Baldessar, M. J.; Spanho, F. J. Seção 2 Gamification e Teoria do Flow. In: FADEL, L.M.; ULBRICHT, V.R.; BATISTA, C.; VANZIN, T. 2014. Gamification na Educação. Editora: Pimenta Cutural. São Paulo.

Fardo, M. F. (2013). A Gamificação Aplicada em Ambientes de Aprendizagem. RenoteNovas Tecnologias na Educação. 11 (1).

Gomes, A. S., Padovani, S. (2005). Usabilidade no ciclo de desenvolvimento de software educativo. In: SBIE Simpósio Brasileiro de Informática na Educação, Juiz de Fora-MG.

Kim, T. J., Lee, W. H. (2013). Dynamical model for gamification of learning (DMGL), Multimedia Tools and Applications, Springer US.

Kitchenham, B., Charters S. Guidelines for performing Systematic Literature Reviews in Software Engineering. Vol 2.3 EBSE Technical Report, EBSE-2007-01, Software Engineering Group, School of Computer Science and Mathematics, Keele University, Keele, UK, (2007).

Lovis, K. A. (2007). Atividades Envolvendo Tipos de Softwares Educacionais. Monografia, Universidade Federal de Santa Catarina.

Morrison, B. B., Disalvo, B. (2014). Khan academy gamifies computer science. In Proceedings of the 45th ACM technical symposium on Computer science education (SIGCSE '14). ACM, New York, NY, USA, 39-44.

Peixoto, M. M. e Silva, C. (2015) Requisitos para Softwares Educacionais Gamificados: Uma Revisão Sistemática de Literatura. In Anais do 18o Ws em Engenharia de Requisitos, Lima, Perú.

Valente, J. A. (1997). O computador na sociedade do conhecimento In. Valente, J. A.(org.) $O$ computador na sociedade do conhecimento. Campinas: NIED/UNICAMP.

Vianna, Y. et. al. (2013). Gamification, Inc : como reinventar empresas a partir de jogos. Editora MJV Press. $1^{\circ}$ Edição. 